\title{
RELATIVE CUBULATIONS AND GROUPS WITH A 2-SPHERE BOUNDARY
}

\author{
DANIEL GROVES AND EDUARD EINSTEIN
}

\begin{abstract}
We introduce a new kind of action of a relatively hyperbolic group on a CAT(0) cube complex, called a relatively geometric action. We provide an application to characterize finite-volume Kleinian groups in terms of action on cube complexes, analogous to the results of Markovic and Haïssinsky in the closed case.
\end{abstract}

\section{INTRODUCTION}

The Cannon Conjecture (see [5, Conjecture 11.34], [6, Conjecture 5.1]) is one of the central problems in geometric group theory. Using the work of Agol [1], Markovic [20, Theorem 1.1] gave an approach to proving the Cannon Conjecture using $\mathrm{CAT}(0)$ cube complexes and quasi-convex codimension 1 surface subgroups. This was slightly generalized by Haïssinsky [14, Theorem 1.10], who proved that a Gromov hyperbolic group whose boundary is a 2-sphere is virtually Kleinian if and only if it acts properly and cocompactly on a CAT(0) cube complex ${ }^{1}$.

A relative version of the Cannon Conjecture states that a relatively hyperbolic group (with abelian parabolic subgroups) whose (Bowditch) boundary is a $2-$ sphere is a Kleinian group (see [19, Problem 57], for example). In [12], it was proved that the Relative Cannon Conjecture is implied by the Cannon Conjecture.

In this paper we introduce a new kind of action of a relatively hyperbolic group on a CAT(0) cube complex, called a relatively geometric action (see Definition 2.1 below). Contrary to proper and cocompact actions, whose coarse geometry is that of the Cayley graph, relatively geometric actions have the coarse geometry of the coned Cayley graph (see Proposition 2.2 below), and hence their geometry can be expected to more faithfully exhibit the relatively hyperbolic geometry of groups.

It follows from the work of Cooper-Futer [8, Theorem 1.1] and the Sageev construction [21] that if $M$ is a finite-volume hyperbolic 3 -manifold then $\pi_{1}(M)$ admits a relatively geometric action on a CAT(0) cube complex (see Theorem 3.1 below).

Applying the results from [12], [11] and [8], we prove the following relative version of Haïssinsky's result.

Theorem 1.1. Suppose that $(G, \mathcal{P})$ is relatively hyperbolic, that the elements of $\mathcal{P}$ are free abelian, and that the (Bowditch) boundary of $(G, \mathcal{P})$ is a $2-$ sphere. Then $G$ is Kleinian if and only if $G$ admits a relatively geometric action on a $C A T(0)$ cube complex.

The second author was partially supported by the National Science Foundation, DMS-1507067.

${ }^{1}$ In fact, Haïssinsky proved this result more generally for hyperbolic groups with planar boundary 


\section{Relative CUBULATIONS}

The theory of hyperbolic groups acting properly and cocompactly on CAT $(0)$ cube complexes is by now well developed (see [23, 13, 17, 3, 11], etc.). In the relatively hyperbolic situation, two generalizations have been previously studied: proper and cocompact actions (as in $[22,23]$ ) and proper and 'cosparse' actions (see [16, 22]).

The following definition provides another condition for relatively hyperbolic groups which restricts to being proper and cocompact in case $G$ is hyperbolic and $\mathcal{P}=\varnothing$.

Definition 2.1. Suppose that $(G, \mathcal{P})$ is a group pair. A (cellular) action of $G$ on a cell complex $X$ is relatively geometric (with respect to $\mathcal{P}$ ) if

(1) $G \backslash X$ is compact;

(2) Each element of $\mathcal{P}$ acts elliptically on $X$; and

(3) Each stabilizer in $G$ of a cell in $X$ is either finite or else conjugate to a finiteindex subgroup of $\mathcal{P}$.

In Section 3 below we give natural examples of relatively geometric actions on $\mathrm{CAT}(0)$ cube complexes, provided by the work of Cooper and Futer [8].

The authors will investigate relatively geometric actions of relatively hyperbolic groups on CAT(0) cube complexes in future work. For the remainder of this section, we record some basic features of relatively geometric actions.

The following is an immediate consequence of [7, Theorem 5.1].

Proposition 2.2. Suppose that $(G, \mathcal{P})$ is relatively hyperbolic, and that $G$ admits a relatively geometric action on a $C A T(0)$ cube complex $X$. Then $X$ is quasi-isometric to the coned-off Cayley graph of $(G, \mathcal{P})$, and consequently is $\delta$-hyperbolic for some $\delta$.

The following is an immediate consequence of [11, Corollary 6.5]. See [11, §6] for the definition of $\mathcal{Q}$-fillings, and more context.

Proposition 2.3. Suppose that $(G, \mathcal{P})$ is relatively hyperbolic and that $G$ admits a relatively geometric action on a $C A T(0)$ cube complex $X$. Let $\mathcal{Q}$ be a collection of finite-index subgroups of elements of $\mathcal{P}$ so that any infinite cell stabilizer contains a conjugate of an element of $\mathcal{Q}$. For sufficiently long $\mathcal{Q}$-fillings

$$
G \rightarrow \bar{G}=G / K
$$

of $(G, \mathcal{P})$, the quotient $K \backslash X$ is a $C A T(0)$ cube complex.

Proposition 2.3 provides one major benefit of relatively geometric actions over proper and cocompact (or proper and cosparse) actions. Namely, if the images of the elements of $\mathcal{P}$ in $G / K$ are hyperbolic and virtually special (for example, finite or virtually cyclic) then [11, Theorem D] implies that $G / K$ is virtually special. This allows one to prove properties of $G$ by taking virtually special hyperbolic Dehn fillings $G / K$ and applying the properties of virtually special hyperbolic groups. This technique is used in the proof of Theorem 1.1 below, and will be crucial in our future work.

Relatively quasi-convex subgroups of relatively hyperbolic groups were investigated in [15]. See that paper for many equivalent definitions, or [2, 10] for yet more equivalent definitions. A relatively quasi-convex subgroup $H$ of a relatively 
hyperbolic group $(G, \mathcal{P})$ is full if for any $g \in G$ and $P \in \mathcal{P}$, the subgroup $H^{g} \cap P$ is either finite, or of finite-index in $P$.

Proposition 2.4. Suppose that $(G, \mathcal{P})$ is relatively hyperbolic and that $\mathcal{H}$ is a finite collection of full relatively quasi-convex codimension 1 subgroups. Then each one-ended element of $\mathcal{P}$ acts elliptically on the cube complex dual to $\mathcal{H}$.

Proof. Let $\mathcal{H}$ be a finite collection of full relatively quasi-convex codimension 1 subgroups of $G$ and let $X$ be a CAT(0) cube complex dual to $\mathcal{H}$ obtained by the Sageev construction. In order to obtain a contradiction, suppose that $P \in \mathcal{P}$ is oneended and that $P$ does not act elliptically on $X$. Then any orbit $P . x$ of $P$ in $X$ is unbounded, and for any such orbit there is a hyperplane $W$ in $X$ so that there are elements of P.x on either side of $W$, arbitrarily far from $W$.

It is straightforward to see that $\operatorname{Stab}(W) \cap P$ is a codimension 1 subgroup of $P$. Since $P$ is one-ended, every codimension 1 subgroup of $P$ is infinite. But $\operatorname{Stab}(W)$ is full so $\operatorname{Stab}(W) \cap P$ is finite-index in $P$. It follows that any orbit $P . x$ is contained in a bounded neighborhood of $W$, contradicting our choice of $W$.

For a hyperbolic group $G$, Sageev [21, Theorem 3.1] proved that the cube complex associated to a finite collection of quasi-convex codimension 1 subgroups is $G$-cocompact. The following is the appropriate relatively geometric version, and follows quickly from results of Hruska-Wise [16].

Proposition 2.5. Suppose that $(G, \mathcal{P})$ is relatively hyperbolic, that each element of $\mathcal{P}$ is one-ended, and that $\mathcal{H}$ is a finite collection of full relatively quasi-convex codimension 1 subgroups. Then the action of $G$ on the cube complex dual to $\mathcal{H}$ is $G$-cocompact.

Proof. The condition that elements of $\mathcal{H}$ are full implies that the cubulation of each element of $\mathcal{P}$ induced by $\mathcal{H}$ (in any variation, see [16]) is a finite cube complex. The result now follows immediately from [16, Theorem 7.12].

The following result is a slight variation of [4, Theorem 5.1], and provides a useful criterion for actions to be relatively geometric.

Theorem 2.6. Let $(G, \mathcal{P})$ be relatively hyperbolic and suppose that for every pair of distinct points $u, v \in \partial(G, \mathcal{P})$ there is a full relatively quasi-convex codimension 1 subgroup $H$ of $G$ so that $u, v$ lie in $H$-distinct components of $\partial G \backslash \Lambda H$. Then there exist finitely many full relatively quasi-convex codimension 1 subgroups of $G$ so that the action of $G$ on the dual cube complex is relatively geometric.

Proof. For any finite collection of full quasi-convex codimension 1 subgroups, the action on the dual cube complex is $G$-cocompact by Proposition 2.5, and elements of $\mathcal{P}$ act elliptically by Proposition 2.4. Therefore, it remains to prove that there is a finite collection of full relatively quasi-convex codimension 1 subgroups with respect to which the stabilizers for the dual cube complex are finite or parabolic. Thus, we need to show that there is a finite collection of such subgroups which 'cut' each loxodromic element of $G$. This can be achieved by applying the proof of [4, Theorem 5.1] directly.

\section{Finite-VOLUME HYPERBOLIC 3-MANIFOLDS AND RELATIVELY GEOMETRIC ACTIONS}

In this section, we explain how the works of Cooper-Futer [8] and BergeronWise [4] together imply the following result. 
Theorem 3.1. Suppose that $M$ is a finite-volume hyperbolic 3-manifold. Then $\pi_{1}(M)$ admits a relatively geometric action on a cube complex.

In case $M$ is closed, this result is due to Bergeron-Wise [4], using work of KahnMarkovic [18]. In the finite-volume case, Cooper and Futer proved that $\pi_{1}(M)$ admits a proper and cocompact action on a CAT( 0 ) cube complex, using $[8$, Theorem 1.2] and the results in [4].

Definition 3.2. [8] A collection of immersed surfaces in a hyperbolic 3-manifold $M$ is ubiquitous if for any pair of hyperbolic planes $\Pi, \Pi^{\prime} \subset \mathbb{H}^{3}$ whose distance $d\left(\Pi, \Pi^{\prime}\right)$ is greater than 0 there is some surface $S$ in the collection with an embedded preimage $\widetilde{S} \subset \mathbb{H}^{3}$ that separates $\Pi$ from $\Pi^{\prime}$.

Theorem 3.3. [8, Theorem 1.1] Let $M$ be a complete, finite-volume hyperbolic 3-manifold. Then the set of closed immersed quasi-Fuchsian surfaces in $M$ is ubiquitous.

Noting that the closed surfaces in Theorem 3.3 contain no parabolics, and so the corresponding subgroups of $\pi_{1}(M)$ are full, Theorem 3.1 is an immediate consequence of Theorems 3.3 and 2.6.

\section{CRiterion FOR Relative CANNON}

For the convenience of the reader, we recall the statement of Theorem 1.1.

Theorem 1.1. Suppose that $(G, \mathcal{P})$ is relatively hyperbolic, that the elements of $\mathcal{P}$ are free abelian, and that the (Bowditch) boundary of $(G, \mathcal{P})$ is a 2 -sphere. Then $G$ is Kleinian if and only if $G$ admits a relatively geometric action on a $C A T(0)$ cube complex.

Proof. Suppose that $G$ is Kleinian. Then $G$ admits a relatively geometric action by Theorem 3.1 above.

Conversely, suppose that $G$ admits a relatively geometric action on a CAT(0) cube complex $X$. Let $\mathcal{Q}$ be as in the statement of Proposition 2.3. According to Proposition 2.3, for sufficiently long $\mathcal{Q}$-fillings $G \rightarrow G / K$ the quotient space $K \backslash X$ is a $\operatorname{CAT}(0)$ cube complex.

We consider sufficiently long co-(virtually cyclic fillings), obtained by choosing cyclic subgroups as filling kernels. ${ }^{2}$ According to [12, Theorem 1.2], for sufficiently long such fillings the quotient $G / K$ is a word-hyperbolic group whose (Gromov) boundary is a 2-sphere. On the other hand, such a $G / K$ acts cocompactly on the CAT(0) cube complex $K \backslash X$, with virtually cyclic cell stabilizers. Since virtually cyclic groups are virtually special, and virtually cyclic subgroups of hyperbolic groups are quasi-convex, it follows from [11, Theorem D] that such a $G / K$ is a virtually special group (and in particular it is cubulable). By [14, Theorem 1.10], any such $G / K$ is virtually Kleinian. In fact, since the parabolic subgroups of $G$ are free abelian, $G$ cannot have a finite normal subgroup. It now follows from [9, Theorem 7.2] that such a $G / K$ has no finite normal subgroup, and hence it acts faithfully on its boundary. Therefore, $G / K$ is Kleinian.

We now take a longer and longer sequence of fillings of this form, obtaining a collection of hyperbolic quotients $G \rightarrow G_{i}=G / K_{i}$ so that each $G_{i}$ is Kleinian.

\footnotetext{
${ }^{2}$ Note that an element $P \in \mathcal{P}$ fixes a point $\xi_{P} \in \partial(G, \mathcal{P})$, and by the dynamical characterization of relatively hyperbolic groups [24] the group $P$ acts properly and cocompactly on $\partial(G, \mathcal{P}) \backslash \xi_{P} \cong \mathbb{R}^{2}$. It follows that each element of $\mathcal{P}$ is free abelian of rank 2 .
} 
As in the proof of $\left[12\right.$, Corollary 1.4] we get a sequence of representations $\rho_{i}: G \rightarrow$ $\operatorname{Isom}\left(\mathbb{H}^{3}\right)$, and exactly as in [12] this sequence must converge to a discrete faithful representation of $G$ into $\operatorname{Isom}\left(\mathbb{H}^{3}\right)$, which shows that $G$ is Kleinian, as required.

\section{REFERENCES}

[1] I. Agol. The virtual Haken conjecture. Doc. Math., 18:1045-1087, 2013. With an appendix by Agol, Daniel Groves, and Jason Manning.

[2] I. Agol, D. Groves, and J. F. Manning. Residual finiteness, QCERF and fillings of hyperbolic groups. Geom. Topol., 13(2):1043-1073, 2009.

[3] I. Agol, D. Groves, and J. F. Manning. An alternate proof of Wise's Malnormal Special Quotient Theorem. Forum of Mathematics, Pi, 4, 2016.

[4] N. Bergeron and D. T. Wise. A boundary criterion for cubulation. Amer. J. Math., 134(3):843-859, 2012.

[5] J. W. Cannon. The theory of negatively curved spaces and groups. In Ergodic theory, symbolic dynamics, and hyperbolic spaces (Trieste, 1989), Oxford Sci. Publ., pages 315-369. Oxford Univ. Press, New York, 1991.

[6] J. W. Cannon and E. L. Swenson. Recognizing constant curvature discrete groups in dimension 3. Trans. Amer. Math. Soc., 350(2):809-849, 1998.

[7] R. Charney and J. Crisp. Relative hyperbolicity and Artin groups. Geom. Dedicata, 129:1-13, 2007.

[8] D. Cooper and D. Futer. Ubiquitous quasi-fuchsian surfaces in cusped hyperbolic 3-manifolds. arXiv.org/abs/1705.02890, 2017.

[9] D. Groves and J. F. Manning. Dehn filling in relatively hyperbolic groups. Israel Journal of Mathematics, 168:317-429, 2008

[10] D. Groves and J. F. Manning. Quasiconvexity and Dehn filling. arXiv.org/abs/1708.07968, 2017.

[11] D. Groves and J. F. Manning. Hyperbolic groups acting improperly. arxiv.org/abs/1808.02325, 2018.

[12] D. Groves, J. F. Manning, and A. Sisto. Boundaries of Dehn fillings. arxiv.org/abs/1612.03497, 2016.

[13] F. Haglund and D. T. Wise. A combination theorem for special cube complexes. Ann. of Math. (2), 176(3):1427-1482, 2012.

[14] P. Haïssinsky. Hyperbolic groups with planar boundaries. Invent. Math., 201(1):239-307, 2015.

[15] G. C. Hruska. Relative hyperbolicity and relative quasiconvexity for countable groups. Algebr. Geom. Topol., 10(3):1807-1856, 2010.

[16] G. C. Hruska and D. T. Wise. Finiteness properties of cubulated groups. Compos. Math., 150(3):453506, 2014.

[17] T. Hsu and D. T. Wise. Cubulating malnormal amalgams. Invent. Math., 199(2):293-331, 2015.

[18] J. Kahn and V. Markovic. Immersing almost geodesic surfaces in a closed hyperbolic three manifold. Ann. of Math. (2), 175(3):1127-1190, 2012.

[19] M. Kapovich. Problems on boundaries of groups and Kleinian groups. https://www. math.ucdavis.edu/ kapovich/EPR/problems.pdf, 2007.

[20] V. Markovic. Criterion for Cannon's conjecture. Geom. Funct. Anal., 23(3):1035-1061, 2013.

[21] M. Sageev. Codimension-1 subgroups and splittings of groups. J. Algebra, 189(2):377-389, 1997.

[22] M. Sageev and D. T. Wise. Cores for quasiconvex actions. Proc. Amer. Math. Soc., 143(7):2731-2741, 2015.

[23] D. T. Wise. The structure of groups with a quasiconvex hierarchy, 2012. Unpublished manuscript.

[24] A. Yaman. A topological characterisation of relatively hyperbolic groups. J. Reine Angew. Math., 566:41-89, 2004.

DePartment of MATHEMATICS, STATISTICS, AND COMPUTER SCIENCE, UNIVERSITY OF ILLINOIS At Chicago, 322 Science and Engineering OfFices (M / C 249), 851 S. Morgan ST., Chicago, IL 60607-7045

E-mail address: groves@math.uic.edu

E-mail address: einstein@uic.edu 